\title{
APPROXIMATION BY WALSH-KACZMARZ-FEJÉR MEANS ON THE HARDY SPACE
}

\author{
GEORGE TEPHNADZE
}

\begin{abstract}
The main aim of this paper is to find necessary and sufficient conditions for the convergence of Walsh-Kaczmarz-Fejér means in the terms of the modulus of continuity on the Hardy spaces $H_{p}$, when $0<p \leq 1 / 2$.
\end{abstract}

\section{INTRODUCTION}

2000 Mathematics Subject Classification. 42C10.

Key words and phrases: Walsh-Kaczmarz system, Fejér means, martingale Hardy space, modulus of continuity.

The a.e. convergence of Walsh-Fejér means $\sigma_{n} f$ was proved by Fine [1]. Yano [35] has proved that $\left\|K_{n}\right\| \leq 2(n \in \mathbb{N})$. Consequently, $\left\|\sigma_{n} f-f\right\|_{p} \rightarrow$ 0 , as $n \rightarrow \infty\left(f \in L_{p}, 1 \leq p \leq \infty\right)$. However (see [13, 19]) the rate of convergence can not be better then $O\left(n^{-1}\right)(n \rightarrow \infty)$ for nonconstant functions. Fridli [2] use dyadic modulus of continuity to characterize the set of functions in the space $L_{p}$, whose Walsh-Fejér means converge at the given rate.

In 1975 Schipp [20] showed that the maximal operator $\sigma^{*}$ is of weak type $(1,1)$ and of type $(p, p)$ for $1<p \leq \infty$. The boundedness fails to hold for $p=1$. But, Fujii [4 proved that $\sigma^{*}$ is bounded from the dyadic Hardy space $H_{1}$ to the space $L_{1}$. The theorem of Fujii was extended by Weisz [33], he showed that the maximal operator $\sigma^{*}$ is bounded from the martingale Hardy space $H_{p}$ to the space $L_{p}$ for $p>1 / 2$. Simon gave a counterexample [22, which shows that the boundedness does not hold for $0<p<1 / 2$. The counterexample for $p=1 / 2$ due to Goginava [9] (see also [26]). In the endpoint case $p=1 / 2$ Weisz [34] proved that $\sigma^{*}$ is bounded from the Hardy space $H_{1 / 2}$ to the space weak- $L_{1 / 2}$.

In 1948 S̆neider 25 introduced the Walsh-Kaczmarz system and showed that the inequality

$$
\limsup _{n \rightarrow \infty} \frac{D_{n}^{\kappa}(x)}{\log n} \geq C>0
$$

holds a.e. In 1974 Schipp [21 and Young [36] proved that the WalshKaczmarz system is a convergence system. In 1981, Skvortsov 24] showed

The research was supported by Shota Rustaveli National Science Foundation grant no.13/06 (Geometry of function spaces, interpolation and embedding theorems). 
that the Fejér means with respect to the Walsh-Kaczmarz system converge uniformly to $f$ for any continuous functions $f$. Gát [5] proved that, for any integrable functions, the Fejér means with respect to the Walsh-Kaczmarz system converge almost everywhere to the function. He showed that the maximal operator of Walsh-Kaczmarz-Fejér means $\sigma^{\kappa, *}$ is weak type $(1,1)$ and of type $(p, p)$ for all $1<p \leq \infty$. Gát's result was generalized by Simon [23], who showed that the maximal operator $\sigma^{\kappa, *}$ is of type $\left(H_{p}, L_{p}\right)$ for $p>1 / 2$. In the endpoint case $p=1 / 2$ Goginava [8] (see also [7, 6]) proved that maximal operator $\sigma^{\kappa, *}$ is not of type $\left(H_{1 / 2}, L_{1 / 2}\right)$ and Weisz [34] showed that the maximal operator is of weak type $\left(H_{1 / 2}, L_{1 / 2}\right)$. In [7] and [29] it is proved that the maximal operator $\widetilde{\sigma}^{\kappa, *}$ defined by

$$
\widetilde{\sigma}_{p}^{\kappa, *}:=\sup _{n \in \mathbb{N}} \frac{\left|\sigma_{n}^{\kappa}\right|}{(n+1)^{1 / p-2} \log ^{2[1 / 2+p]}(n+1)},
$$

where $0<p \leq 1 / 2$ and $[1 / 2+p]$ denotes integer part of $1 / 2+p$, is bounded from the Hardy space $H_{p}$ to the space $L_{p}$. As a corollary we get

$$
\left\|\sigma_{n}^{\kappa} f\right\|_{p} \leq c_{p}(n+1)^{1 / p-2} \log ^{2[1 / 2+p]}(n+1)\|f\|_{H_{p}} .
$$

Moreover, for any nondecreasing function $\varphi: \mathbb{N}_{+} \rightarrow[1, \infty)$ satisfying the condition

$$
\varlimsup_{n \rightarrow \infty} \frac{(n+1)^{1 / p-2} \log ^{2[1 / 2+p]}(n+1)}{\varphi(n)}=+\infty,
$$

there exists a martingale $f \in H_{p}$, such that

$$
\sup _{n \in \mathbb{N}}\left\|\frac{\sigma_{n}^{\kappa} f}{\varphi(n)}\right\|_{p}=\infty .
$$

For Walsh system analogical theorems are proved in [10], [27] and [28].

It is well-known (see 31]) that

$$
\left\|\sigma_{2^{n}}^{\kappa}(f)-f\right\|_{H_{p}} \rightarrow 0, \text { when } n \rightarrow \infty, \quad\left(f \in H_{p}, p>0\right) .
$$

Móricz and Siddiqi [14] investigates the approximation properties of some special Nörlund means of Walsh-Fourier series of $L_{p}$ function in norm. Fridli, Manchanda and Siddiqi [3] improved and extended the results of Móricz and Siddiqi [14] among them in $H_{p}$ norm, where $0<p<1$. In [12] Goginava investigated the behavior of Cesáro means of Walsh-Fourier series in detail. Analogical results for Walsh-Kaczmarz-Fourier series were proved by Nagy in [15, 16, 17, 18.

The main aim of this paper is to find necessary and sufficient conditions for the convergence of Fejér means in the terms of the modulus of continuity on the Hardy spaces $H_{p}$, when $0<p<1 / 2$ and $p=1 / 2$, respectively. That is, the most investigated end point case $p=1 / 2$ is also discussed.

The paper is organized as following: In Section 3 we present and discuss the main results and in Section 4 the auxiliary propositions will be given. In Section 5 the the proofs can be found. Moreover, in order not to disturb 
our discussions in these Sections some definitions and notations are given in Section 2.

\section{Definitions and Notations}

Now, we give a brief introduction to the theory of dyadic analysis [19]. Let $\mathbb{N}_{+}$denote the set of the positive integers, $\mathbb{N}:=\mathbb{N}_{+} \cup\{0\}$. Denote $\mathbb{Z}_{2}$ the discrete cyclic group of order 2 , that is $\mathbb{Z}_{2}=\{0,1\}$, where the group operation is the modulo 2 addition and every subset is open. The Haar measure on $\mathbb{Z}_{2}$ is given such that the measure of a singleton is $1 / 2$. Let $G$ be the complete direct product of the countable infinite copies of the compact groups $\mathbb{Z}_{2}$. The elements of $G$ are of the form $x=\left(x_{0}, x_{1}, \ldots, x_{k}, \ldots\right)$ with $x_{k} \in\{0,1\}(k \in \mathbb{N})$. The group operation on $G$ is the coordinatewise addition, the measure (denoted by $\mu$ ) and the topology are the product measure and topology. The compact Abelian group $G$ is called the Walsh group. A base for the neighborhoods of $G$ can be given in the following way:

$$
\begin{aligned}
& I_{0}(x):=G, \\
& I_{n}(x):=I_{n}\left(x_{0}, \ldots, x_{n-1}\right):=\left\{y \in G: y=\left(x_{0}, \ldots, x_{n-1}, y_{n}, y_{n+1}, \ldots\right)\right\},
\end{aligned}
$$

where $x \in G$ and $n \in \mathbb{N}$. These sets are called dyadic intervals. Let $0=$ $(0: i \in \mathbb{N}) \in G$ denote the null element of $G$, and $I_{n}:=I_{n}(0)(n \in \mathbb{N})$. Set $e_{n}:=\left(0, \ldots, 0, x_{n}=1,0, \ldots\right) \in G$ and

$J_{N}^{m, l}:=I_{N}\left(x_{0}, \ldots, x_{m}=1,0, \ldots, x_{l}=1,0, \ldots, 0\right), \quad l=0, \ldots, N-1, \quad m=-1, \ldots, l$.

For $k \in \mathbb{N}$ and $x \in G$ denote

$$
r_{k}(x):=(-1)^{x_{k}}
$$

the $k$-th Rademacher function.

If $n \in \mathbb{N}$, then $n=\sum_{i=0}^{\infty} n_{i} 2^{i}$ can be written, where $n_{i} \in\{0,1\} \quad(i \in \mathbb{N})$, i.

e. $n$ is expressed in the number system of base 2 . Denote $|n|:=\max \{j \in$ $\left.\mathbb{N}: n_{j} \neq 0\right\}$, that is $2^{|n|} \leq n<2^{|n|+1}$.

The Walsh-Paley system is defined as the sequence of Walsh-Paley functions:

$$
w_{n}(x):=\prod_{k=0}^{\infty}\left(r_{k}(x)\right)^{n_{k}}=r_{|n|}(x)(-1) \sum_{k=0}^{|n|-1} n_{k} x_{k} \quad(x \in G, n \in \mathbb{N}) .
$$

The Walsh-Kaczmarz functions are defined by $\kappa_{0}=1$ and for $n \geq 1$

$$
\kappa_{n}(x):=r_{|n|}(x) \prod_{k=0}^{|n|-1}\left(r_{|n|-1-k}(x)\right)^{n_{k}}=r_{|n|}(x)(-1) \sum_{k=0}^{|n|-1} n_{k} x_{|n|-1-k} .
$$

Skvortsov (see [24]) gave a relation between the Walsh-Kaczmarz functions and Walsh-Paley functions by the of the transformation $\tau_{A}: G \rightarrow G$ defined by 


$$
\tau_{A}(x):=\left(x_{A-1}, x_{A-2}, \ldots, x_{1}, x_{0}, x_{A}, x_{A+1}, \ldots\right),
$$

for $A \in \mathbb{N}$. By the definition we have

$$
\kappa_{n}(x)=r_{|n|}(x) w_{n-2^{|n|}}\left(\tau_{|n|}(x)\right), \quad(n \in \mathbb{N}, x \in G) .
$$

The Dirichlet kernels are defined

$$
D_{n}^{\alpha}:=\sum_{k=0}^{n-1} \alpha_{k}
$$

where $\alpha_{n}=w_{n}$ or $\kappa_{n}(n \in \mathbb{N}), D_{0}^{\alpha}:=0$. the $2^{n}$-th Dirichlet kernels have a closed form (see e.g. [19])

$$
D_{2^{n}}^{w}(x)=D_{2^{n}}^{\kappa}(x)=D_{2^{n}}(x)= \begin{cases}2^{n} & \text { if } x \in I_{n} \\ 0 & \text { if } x \notin I_{n}\end{cases}
$$

The norm (or quasi-norm) of the space $L_{p}(G)$ is defined by

$$
\|f\|_{p}:=\left(\int_{G}|f|^{p} d \mu\right)^{1 / p} \quad(0<p<\infty) .
$$

The space $L_{p, \infty}(G)$ consists of all measurable functions $f$ for which

$$
\|f\|_{L_{p, \infty}}:=\sup _{\lambda>0} \lambda \mu\{|f|>\lambda\}^{1 / p} \leq c<\infty .
$$

The $\sigma$-algebra generated by the dyadic intervals of measure $2^{-k}$ will be denoted by $F_{k}(k \in \mathbb{N})$. Denote by $f=\left(f^{(n)}, n \in \mathbb{N}\right)$ a martingale with respect to $\left(F_{n}, n \in \mathbb{N}\right.$ ) (for details see, e. g. [31]). The maximal function of a martingale $f$ is defined by

$$
f^{*}=\sup _{n \in \mathbb{N}}\left|f^{(n)}\right| .
$$

In case $f \in L_{1}(G)$, the maximal function can also be given by

$$
f^{*}(x)=\sup _{n \in \mathbb{N}} \frac{1}{\mu\left(I_{n}(x)\right)}\left|\int_{I_{n}(x)} f(u) d \mu(u)\right|, \quad x \in G .
$$

For $0<p<\infty$ the Hardy martingale space $H_{p}(G)$ consists of all martingales for which

$$
\|f\|_{H_{p}}:=\left\|f^{*}\right\|_{p}<\infty
$$

The best approximation of $f \in L_{p}(G)(1 \leq p \in \infty)$ is defined as

$$
E_{n}\left(f, L_{p}\right)=\inf _{\psi \in p_{n}}\|f-\psi\|_{p}
$$

where $p_{n}$ is set of all Walsh-Kaczmarz polynomials of order less than $n \in \mathbb{N}$. 
The integrated modulus of continuity of the function $f \in L_{p}(G)$, is defined by

$$
\omega_{p}\left(1 / 2^{n}, f\right)=\sup _{h \in I_{n}}\|f(\cdot+h)-f(\cdot)\|_{p} .
$$

The concept of modulus of continuity in $H_{p}(G)(0<p \leq 1)$ is defined in the following way

$$
\omega_{H_{p}}\left(1 / 2^{n}, f\right):=\left\|f-S_{2^{n}} f\right\|_{H_{p}} .
$$

Since $\|f\|_{H_{p}} \sim\|f\|_{p}$, when $p>1$, we obtain that

$$
\omega_{H_{p}}\left(1 / 2^{n}, f\right) \sim\left\|f-S_{2^{n}} f\right\|_{p}, \quad p>1 .
$$

On the ather hand Watari [30] showed that there are strong connection among $\omega_{p}\left(1 / 2^{n}, f\right), E_{2^{n}}\left(f, L_{p}\right)$ and $\left\|f-S_{2^{n}} f\right\|_{p}, \quad p \geq 1, n \in \mathbb{N}$. In particular

$$
\omega_{p}\left(1 / 2^{n}, f\right) / 2 \leq\left\|f-S_{2^{n}} f\right\|_{p} \leq \omega_{p}\left(1 / 2^{n}, f\right)
$$

and

$$
\left\|f-S_{2^{n}} f\right\|_{p} / 2 \leq E_{2^{n}}\left(f, L_{p}\right) \leq\left\|f-S_{2^{n}} f\right\|_{p} .
$$

If $f \in L_{1}(G)$, then it is easy to show that the sequence $\left(S_{2^{n}} f: n \in \mathbb{N}\right)$ is a martingale. If $f$ is a martingale, that is $f=\left(f^{(0)}, f^{(1)}, \ldots\right)$ then the Walsh(Kaczmarz)-Fourier coefficients must be defined in a little bit different way:

$$
\widehat{f}(i)=\lim _{k \rightarrow \infty} \int_{G} f^{(k)}(x) \alpha_{i}(x) d \mu(x), \quad\left(\alpha_{i}=w_{i} \text { or } \kappa_{i}\right) .
$$

The Walsh-(Kaczmarz)-Fourier coefficients of $f \in L_{1}(G)$ are the same as the ones of the martingale $\left(S_{2^{n}} f: n \in \mathbb{N}\right)$ obtained from $f$.

The partial sums and Fejér means of the Walsh-(Kaczmarz)-Fourier v of martingale $f \in H_{p}$ are defined by

$$
S_{n}^{\alpha}(f):=\sum_{i=0}^{n-1} \widehat{f}(i) \alpha_{i}, \quad \sigma_{n}^{\alpha}(f)=\frac{1}{n} \sum_{j=1}^{n} S_{j}^{\alpha}(f), \quad(\alpha=w \text { or } \kappa)
$$

respectively.

The Fejér kernel of order $n$ of the Walsh-(Kaczmarz)-Fourier series defined by

$$
K_{n}^{\alpha}:=\frac{1}{n} \sum_{k=1}^{n} D_{k}^{\alpha}, \quad(\alpha=w \text { or } \kappa) .
$$

A bounded measurable function $a$ is p-atom, if there exists an interval $I$, such that

$$
\int_{I} a d \mu=0, \quad\|a\|_{\infty} \leq \mu(I)^{-1 / p}, \quad \operatorname{supp}(a) \subset I .
$$


For the martingale $f=\sum_{n=0}^{\infty}\left(f_{n}-f_{n-1}\right)$ the conjugate transforms are defined as

$$
\widetilde{f^{(t)}}=\sum_{n=0}^{\infty} r_{n}(t)\left(f_{n}-f_{n-1}\right),
$$

where $t \in G$ is fixed. Note that $\widetilde{f^{(0)}}=f$. As is well known (see [31])

$$
\left\|\widetilde{f^{(t)}}\right\|_{H_{p}}=\|f\|_{H_{p}}, \quad\|f\|_{H_{p}}^{p} \sim \int_{G}\left\|\widetilde{f^{(t)}}\right\|_{p}^{p} d t, \quad \widetilde{\sigma_{n}^{\kappa} f^{(t)}}=\widetilde{\sigma_{n}^{\kappa}} \widetilde{f^{(t)}} .
$$

3. Formulation of Main Results

Theorem 1. a) Let $0<p<1 / 2$ and

$$
\omega_{H_{p}}\left(1 / 2^{k}, f\right)=o\left(1 / 2^{k(1 / p-2)}\right) \text {, as } k \rightarrow \infty \text {. }
$$

Then

$$
\left\|\sigma_{n}^{\kappa}(f)-f\right\|_{H_{p}} \rightarrow 0, \text { when } n \rightarrow \infty .
$$

b) For any $p \in(0,1 / 2)$ there exists a martingale $f \in H_{p}(G)$ for which

$$
\omega_{H_{p}}\left(1 / 2^{k}, f\right)=O\left(1 / 2^{k(1 / p-2)}\right) \text {, as } k \rightarrow \infty \text {. }
$$

and

$$
\left\|\sigma_{n}^{\kappa}(f)-f\right\|_{L_{p, \infty}} \nrightarrow 0, \text { as } n \rightarrow \infty .
$$

Theorem 2. a) Let

$$
\omega_{H_{1 / 2}}\left(1 / 2^{k}, f\right)=o\left(1 / k^{2}\right), \quad \text { as } k \rightarrow \infty .
$$

Then

$$
\left\|\sigma_{n}^{\kappa}(f)-f\right\|_{H_{1 / 2}} \rightarrow 0, \text { when } n \rightarrow \infty .
$$

b) There exists a martingale $f \in H_{1 / 2}(G)$, for which

$$
\omega_{H_{1 / 2}}\left(1 / 2^{k}, f\right)=O\left(1 / k^{2}\right), \text { as } k \rightarrow \infty .
$$

and

$$
\left\|\sigma_{n}(f)-f\right\|_{1 / 2} \nrightarrow 0 \text { as } n \rightarrow \infty .
$$

\section{AUXILIARY PROPOSITIONS}

Lemma 1. 31] A martingale $f=\left(f_{n}, n \in \mathbb{N}\right)$ is in $H_{p}(0<p \leq 1)$ if and only if there exists a sequence $\left(a_{k}, k \in \mathbb{N}\right)$ of $p$-atoms and a sequence $\left(\mu_{k}, k \in \mathbb{N}\right)$ of real numbers such that for every $n \in \mathbb{N}$

$$
\sum_{k=0}^{\infty} \mu_{k} S_{M_{n}} a_{k}=f_{n}, \quad \text { a.e. }
$$


and

$$
\sum_{k=0}^{\infty}\left|\mu_{k}\right|^{p}<\infty
$$

Moreover, $\|f\|_{H_{p}} \backsim \inf \left(\sum_{k=0}^{\infty}\left|\mu_{k}\right|^{p}\right)^{1 / p}$, where the infimum is taken over all decomposition of $f$ of the form (9).

Lemma 2. [1] Let $2<A \in \mathbb{N}$ and $q_{A}=2^{2 A}+2^{2 A-2}+\ldots+2^{2}+2^{0}$. Then

$$
q_{A-1}\left|K_{q_{A-1}}^{w}(x)\right| \geq 2^{2 m+2 s-3},
$$

for $x \in I_{2 A}\left(0, \ldots, 0, x_{2 m}=1,0 \ldots, 0, x_{2 s}=1, x_{2 s+1}, \ldots, x_{2 A-1}\right), m=0,1, \ldots, A-$ $3, s=m+2, m+3, \ldots, A-1$.

\section{Proof of the Theorems}

Proof of Theorem 1. Let $0<p<1 / 2$. Combining (11) and (5) we conclude that

$$
\begin{aligned}
& \left\|\sigma_{n}^{\kappa} f\right\|_{H_{p}}^{p}=\int_{G}\left\|\widetilde{\sigma_{n}^{\kappa} f^{(t)}}\right\|_{p}^{p} d t \\
= & \int_{G}\left\|\widetilde{\sigma_{n}^{\kappa}} \widetilde{f^{(t)}}\right\|_{p}^{p} d t \leq c_{p} n^{1-2 p} \int_{G}\|\widetilde{f(t)}\|_{H_{p}}^{p} d t \\
\sim & c_{p} n^{1-2 p} \int_{G}\|f\|_{H_{p}}^{p} d t=c_{p} n^{1-2 p}\|f\|_{H_{p}}^{p} .
\end{aligned}
$$

Let $2^{m}<n \leq 2^{m+1}$. Using (10) we have that

$$
\begin{aligned}
& \left\|\sigma_{n}^{\kappa} f-f\right\|_{H_{p}}^{p} \\
\leq & \left\|\sigma_{n}^{\kappa} f-\sigma_{n}^{\kappa} S_{2^{m}} f\right\|_{H_{p}}^{p}+\left\|\sigma_{n}^{\kappa} S_{2^{m}} f-S_{2^{m}} f\right\|_{H_{p}}^{p}+\left\|S_{2^{m}} f-f\right\|_{H_{p}}^{p} \\
= & \left\|\sigma_{n}^{\kappa}\left(S_{2^{m}} f-f\right)\right\|_{H_{p}}^{p}+\left\|S_{2^{m}} f-f\right\|_{H_{p}}^{p}+\left\|\sigma_{n}^{\kappa} S_{2^{m}} f-S_{2^{m}} f\right\|_{H_{p}}^{p} \\
\leq & c_{p}\left(n^{1-2 p}+1\right) \omega_{H_{p}}^{p}\left(1 / 2^{m}, f\right)+\left\|\sigma_{n}^{\kappa} S_{2^{m}} f-S_{2^{m}} f\right\|_{H_{p}}^{p} .
\end{aligned}
$$

By simple calculation we get that

$$
\begin{aligned}
& \sigma_{n^{\kappa}}^{\kappa} S_{2^{m}} f-S_{2^{m}} f=\frac{2^{m}}{n}\left(S_{2^{m}} \sigma_{2^{m}} f-S_{2^{m}} f\right) \\
= & \frac{2^{m}}{n} S_{2^{m}}\left(\sigma_{2^{m}} f-f\right) .
\end{aligned}
$$

Let $p>0$. By applying (3) we obtain that

$$
\begin{aligned}
& \left\|\sigma_{n}^{\kappa} S_{2^{m}} f-S_{2^{m}} f\right\|_{H_{p}}^{p} \\
\leq & \frac{2^{p m}}{n^{p}}\left\|S_{2^{m}}\left(\sigma_{2^{m}} f-f\right)\right\|_{H_{p}}^{p} \\
\leq & \left\|\sigma_{2^{m}} f-f\right\|_{H_{p}}^{p} \rightarrow 0, \text { when } k \rightarrow \infty .
\end{aligned}
$$


It follows that if

$$
\omega_{H_{p}}\left(1 / 2^{m}, f\right)=o\left(1 / 2^{m(1 / p-2)}\right), \text { as } m \rightarrow \infty .
$$

then

$$
\left\|\sigma_{n}^{\kappa} f-f\right\|_{H_{p}} \rightarrow 0, \text { when } n \rightarrow \infty .
$$

Now, we are ready to prove second part of Theorem 1 . We set

$$
a_{i}(x)=2^{i(1 / p-1)}\left(D_{2^{i+1}}(x)-D_{2^{i}}(x)\right),
$$

and

$$
f^{(n)}(x)=\sum_{i=0}^{n} \frac{1}{2^{(1 / p-2) i}} a_{i}(x), n \in \mathbb{N} .
$$

It is easy to show that $f=\left(f^{(1)}, f^{(2)}, \ldots, f^{(n)}, \ldots\right) \in H_{p}$. Indeed, since

$$
\begin{aligned}
& S_{2^{A}} a_{i}(x)= \begin{cases}a_{i}(x), & i<A, \\
0, & i \geq A,\end{cases} \\
& \operatorname{supp}\left(a_{i}\right)=I_{i}, \quad \int_{I_{i}} a_{i} d \mu=0
\end{aligned}
$$

and

$$
\left\|a_{i}\right\|_{\infty} \leq 2^{(1 / p-1) i} \cdot 2^{i}=2^{i / p}=\left(\operatorname{supp} a_{i}\right)^{-1 / p},
$$

if we apply Lemma 1 we conclude that $f \in H_{p}$.

It is easy to show that

$$
\begin{aligned}
& f-S_{2^{n}} f \\
= & \left(f^{(1)}-S_{2^{n}} f^{(1)}, \ldots, f^{(n)}-S_{2^{n}} f^{(n)}, \ldots, f^{(n+k)}-S_{2^{n}} f^{(n+k)}\right) \\
= & \left(0, \ldots, 0, f^{(n+1)}-f^{(n)}, \ldots, f^{(n+k)}-f^{(n)}, \ldots\right) \\
= & \left(0, \ldots, 0, \sum_{i=n}^{n+k} \frac{a_{i}(x)}{2^{i(1 / p-2)}}, \ldots\right), k \in \mathbb{N}_{+} .
\end{aligned}
$$

is a martingale. By using (13) we get that

$$
\begin{gathered}
\omega_{H_{p}}\left(1 / 2^{n}, f\right) \\
\leq \sum_{i=n+1}^{\infty} 1 / 2^{(1 / p-2) i} \leq c / 2^{(1 / p-2) n}=O\left(1 / 2^{(1 / p-2) n}\right) .
\end{gathered}
$$

It is easy to show that

$$
\widehat{f}(j)=2^{i}, \quad \text { if } j \in\left\{2^{i}, \ldots, 2^{i+1}-1\right\}, i=0,1, \ldots
$$


By applying (3) and (14) we have that

$$
\begin{aligned}
& \limsup _{n \rightarrow \infty}\left\|\sigma_{2^{n}+1}^{\kappa}(f)-f\right\|_{L_{p, \infty}} \\
= & \limsup _{n \rightarrow \infty}\left\|\frac{2^{n} \sigma_{2^{n}}^{\kappa}(f)}{2^{n}+1}+\frac{S_{2^{n}}(f)}{2^{n}+1}+\frac{2^{n} \kappa_{2^{n}}}{2^{n}+1}-\frac{2^{n} f}{2^{n}+1}-\frac{f}{2^{n}+1}\right\|_{L_{p, \infty}} \\
\geq & \limsup _{n \rightarrow \infty} \frac{2^{n}}{2^{n}+1}\left\|\kappa_{2^{n}}\right\|_{L_{p, \infty}} \\
& -\limsup _{n \rightarrow \infty} \frac{2^{n}}{2^{n}+1}\left\|\sigma_{2^{n}}^{\kappa}(f)-f\right\|_{L_{p, \infty}} \\
& -\limsup _{n \rightarrow \infty} \frac{1}{2^{n}+1}\left\|S_{2^{n}}(f)-f\right\|_{L_{p, \infty}} \\
> & \limsup _{n \rightarrow \infty} \frac{2^{n}}{2^{n}+1}-o(1)>0 .
\end{aligned}
$$

Theorem 1 is proved.

Proof of Theorem 2. Analogously of first part of Theorem 1 we can show that if modulus of continuity of the martingale $f \in H_{1 / 2}$ satisfies the condition (8), then $\left\|\sigma_{n}^{\kappa} f-f\right\|_{H_{1 / 2}} \rightarrow \infty$, when $n \rightarrow \infty$.

Now, we are ready to prove the second part of theorem 1 . We set

$$
a_{i}(x)=2^{2^{i}}\left(D_{2^{2^{i}+1}}(x)-D_{2^{2^{i}}}(x)\right)
$$

and

$$
f^{(n)}(x)=\sum_{i=1}^{n} \frac{a_{i}(x)}{2^{2 i}} .
$$

It is easy to show that $f=\left(f^{(1)}, f^{(2)}, \ldots, f^{(n)}, \ldots\right) \in H_{1 / 2}$. Indeed, since

$$
\begin{gathered}
S_{2^{A}} a_{i}(x)= \begin{cases}a_{i}(x), & 2^{i}<A, \\
0, & 2^{i} \geq A\end{cases} \\
\operatorname{supp}\left(a_{i}\right)=I_{2^{i}}, \quad \int_{I_{2^{i}}} a_{i} d \mu=0, \quad\left\|a_{i}\right\|_{\infty} \leq\left(2^{2^{i}}\right)^{2}=\left(\mu\left(\operatorname{supp} a_{i}\right)\right)^{2},
\end{gathered}
$$

if we apply Lemma 1 we conclude that $f \in H_{1 / 2}$.

By simple calculation we get that

$$
\omega_{H_{1 / 2}}\left(1 / 2^{n}, f\right) \leq \sum_{i=\left[\log _{2}^{n}\right]}^{\infty} \frac{1}{2^{2 i}}=O\left(1 / n^{2}\right),
$$

where $\left[\log _{2}^{n}\right]$ denotes the integer part of $\log _{2}^{n}$. Hence

$$
\begin{aligned}
& \left\|\sigma_{q_{2 k-1}}^{\kappa}(f)-f\right\|_{1 / 2} \\
& =\left\|\frac{2^{2^{k}} \sigma_{2^{2^{k}}}^{\kappa}(f)}{q_{2^{k-1}}}+\frac{1}{q_{2^{k-1}}} \sum_{j=2^{2^{k}}+1}^{q_{2^{k-1}}} S_{j}^{\kappa} f-\frac{2^{2^{k}} f}{q_{2^{k-1}}}-\frac{q_{2^{k-1}-1} f}{q_{2^{k-1}}}\right\|_{1 / 2}
\end{aligned}
$$

It is easy to show that 


$$
\widehat{f}(j)= \begin{cases}\frac{2^{2^{i}}}{2^{2 i}}, & \text { if } j \in\left\{2^{2^{i}}, \ldots, 2^{2^{i}+1}-1\right\}, i=0,1, \ldots \\ 0, & \text { if } j \notin \bigcup_{n=0}^{\infty}\left\{2^{2^{n}}, \ldots, 2^{2^{n}+1}-1\right\}\end{cases}
$$

Let $2^{2^{i}}<j \leq q_{2^{i-1}}$. Since

$$
D_{s+2^{2^{i}}}^{\kappa}=D_{2^{2^{i}}}+r_{2^{i}} D_{s}^{w}\left(\tau_{2^{i}}\right), \quad \text { when } s<2^{2^{i}}
$$

by applying (16) we obtain that

$$
\begin{aligned}
S_{j}^{\kappa} f & =S_{2^{2^{i}}} f+\sum_{v=2^{2^{i}}}^{j-1} \widehat{f}(v) \kappa_{v}=S_{2^{2^{i}}} f+\frac{2^{2^{i}}}{2^{2 i}} \sum_{v=2^{i}}^{j-1} \kappa_{v} \\
& =S_{2^{2^{i}}} f+\frac{2^{2^{i}}}{2^{2 i}}\left(D_{j}^{\kappa}-D_{2^{2^{i}}}\right)=S_{2^{2^{i}}} f+\frac{2^{2^{i}} r_{2^{i}} D_{j-2^{2^{i}}}^{w}\left(\tau_{2^{i}}\right)}{2^{2 i}} .
\end{aligned}
$$

Hence

$$
\begin{aligned}
& \sum_{j=2^{2^{i}}+1}^{q_{2^{i-1}}} S_{j}^{\kappa} f=\frac{q_{2^{i-1}-1} S_{2^{2^{i}}} f}{q_{2^{i-1}}}+\frac{2^{2^{i}} r_{2^{i}}}{q_{2^{i}} 2^{2 i}} \sum_{j=2^{2^{i}}+1}^{q_{2^{i-1}}} D_{j-2^{2^{k}}}^{w}\left(\tau_{2^{i}}\right) \\
= & \frac{q_{2^{i-1}-1} S_{2^{2^{i}}} f}{q_{2^{i-1}}}+\frac{2^{2^{i}} r_{2^{i}}}{q_{2^{i-1} 2^{2 i}} \sum_{j=1}^{q_{2^{i-1}-1}} D_{j}^{w}\left(\tau_{2^{i}}\right)} \\
= & \frac{q_{2^{i-1}-1} S_{2^{i}} f}{q_{2^{i-1}}}+\frac{2^{2^{i}} r_{2^{i}} q_{2^{i-1}-1} K_{q_{2^{i-1}-1}^{w}}^{w}\left(\tau_{2^{i}}\right)}{q_{2^{i-1}} 2^{2 i}} .
\end{aligned}
$$

By using (15) we get that

$$
\begin{aligned}
& \left\|\sigma_{q_{2^{i-1}}}^{\kappa}(f)-f\right\|_{1 / 2}^{1 / 2} \\
\geq & \frac{c}{2^{i}}\left\|q_{2^{i-1}-1} K_{q_{2^{i-1}-1}}^{w}\left(\tau_{2^{i}}\right)\right\|_{1 / 2}^{1 / 2}-\left(\frac{2^{2^{i}}}{q_{2^{i-1}}}\right)^{1 / 2}\left\|\sigma_{2^{2^{i}}} f-f\right\|_{1 / 2}^{1 / 2} \\
& -\left(\frac{q_{2^{i-1}-1}}{q_{2^{i-1}}}\right)^{1 / 2}\left\|S_{2^{2^{i}}} f-f\right\|_{1 / 2}^{1 / 2}=I V-V-V I .
\end{aligned}
$$

Let $x \in J_{2^{i}}^{2^{i}-2 s-1,2^{i}-2 \eta-1}$, where $\eta=1, \ldots, 2^{i}-3, s=\eta+3, \ldots, 2^{i}-1$. By applying Lemma 2 we have that

$$
q_{2^{i-1}-1}\left|K_{q_{2^{i-1}-1}}^{w}\left(\tau_{2^{i}}(x)\right)\right| \geq 2^{2 \eta+2 s-3} .
$$

We can write 


$$
\begin{aligned}
& \int_{G}\left|q_{2^{i-1}-1} K_{q_{2^{i-1}-1}}^{w}\left(\tau_{2^{i}}(x)\right)\right|^{1 / 2} d \mu(x) \\
\geq & c \sum_{\eta=1}^{2^{i}-3} \sum_{s=\eta+2}^{2^{i}-1} \sum_{x_{2 s+1}=0}^{1} \ldots \sum_{x_{2^{i}-1}=0}^{1} \int_{J_{2^{i}}^{2^{i}-2 s-1,2^{i}-2 \eta-1}}\left|q_{2^{i-1}-1} K_{q_{2^{i-1}-1}}^{w}\left(\tau_{2^{i}}(x)\right)\right|^{1 / 2} d \mu(x) \\
\geq & c \sum_{\eta=1}^{2^{i}-3} \sum_{s=\eta+2}^{2^{i}-1} \frac{1}{2^{2 s}} \sqrt{2^{2 \eta+2 s}} \geq \sum_{\eta=1}^{2^{i}-3} \sum_{s=\eta+2}^{2^{i}-1} \sqrt{\frac{2^{2 \eta}}{2^{2 s}}} \geq c \sum_{\eta=1}^{2^{i}-3} \geq c 2^{i} .
\end{aligned}
$$

By using (17) we have that

$$
\limsup _{i \rightarrow \infty}\left\|\sigma_{q_{2^{i-1}}}^{\kappa}(f)-f\right\|_{1 / 2} \geq c>0 .
$$

Theorem 2 is proved.

Acknowledgment: The author would like to thank the referees for their helpful suggestions.

\section{REFERENCES}

[1] J. Fine: Cesàro summability of Walsh-Fourier series, Proc. Nat. Acad. Sci. USA 41 (1955), 558-591.

[2] S. Fridli: On the rate of convergence of Cesáro means of Walsh-Fourier series, J. Approximation Theory, 76 (1994), no. 1, 31-53.

[3] S. Fridli, P. Manchuda and A. Siddiqi. Approximation by Walsh-Nörlund means, Acta Sci. Math. (Szeged) 74, 3-4, (2008), 593-608.

[4] N. Fujii: A maximal inequality for $H^{1}$-functions on a generalized Walsh-Paley group, Proc. Amer. Math. Soc. 77 (1979), 111-116.

[5] G. Gát: On $(C, 1)$ summability of integrable functions with respect to the WalshKaczmarz system, Studia Math. 130 (2) (1998), 139-148.

[6] G. Gát, U. Goginava, K. Nagy: On the Marcinkiewicz-Fejér means of double Fourier series with respect to the Walsh-Kaczmarz system, Studia Sci. Math. Hungarica 46 (3) (2009), 399-421.

[7] U. Goginava and K. Nagy: On the maximal operator of Walsh-Kaczmarz-Fejer means, Czechoslovak Math. J. 61(136), 2011, 673-686.

[8] U. Goginava: The maximal operator of the Fejér means of the character system of the $p$-series field in the Kaczmarz rearrangement. Publ. Math. Debrecen 71 (2007), no. $1-2,43-55$.

[9] U. Goginava: The maximal operator of the Marcinkiewicz-Fejér means of the ddimensional Walsh-Fourier series, East J. Approx. 12 (2006), no. 3, 295-302.

[10] U. Goginava: Maximal operators of Fejér-Walsh means. Acta Sci. Math. (Szeged) 74 (2008), 615-624.

[11] U. Goginava: Maximal operator of the Fejér means of double Walsh-Fourier series, Acta Math. Hungar, 115 (2007), 333-340.

[12] U. Goginava: On the approximation properties of Cesáro means of negative order of Walsh-Fourier series. Journal of approximation theory 115, 9-20 (2002).

[13] I. Joō, On some problems of M. Horváth, Ann. Univ. Sci. Budapest Sect. Math., 31 (1988), 243-260.

[14] F. Móricz, A. Siddiqi: Approximation by Nörlund means of Walsh-Fourier series. Journal of approximation theory. 70 1992, (375-389). 
[15] K. Nagy: Approximation by Cesáro means of negative order of Walsh-KaczmarzFourier series, East J. Approx., 16 (2010), no. 3, 297-311.

[16] K. Nagy: Approximation by Nörlund means of Walsh-Kaczmarz-Fourier series. Georgian Math. J. 18 (2011), no. 1, 147-162.

[17] K. Nagy: Approximation by Nörlund means of quadratical partial sums of double Walsh-Fourier series, Anal. Math., 36 (2010), no. 4, 299-319.

[18] K. Nagy: Approximation by Nörlund means of double Walsh-Fourier series for Lipschitz functions, Math. Inequal. Appl., 15 (2012), no. 2, 301-322.

[19] F. Schipp, W. R. Wade, P. Simon, and J. Pál: Walsh Series. An Introduction to Dyadic Harmonic Analysis, Adam Hilger (Bristol-New York 1990).

[20] F. Schipp: Certain rearrengements of series in the Walsh series, Mat. Zametki 18 (1975), 193-201.

[21] F. Schipp: Pointwise convergence of expansions with respect to certain product systems, Anal. Math. 2 (1976), 65-76.

[22] P. Simon: Cesàro summability with respect to two-parameter Walsh-system, Monatsh. Math. 131 (2000), 321-334.

[23] P. Simon: On the Cesàro summability with respect to the Walsh-Kaczmarz system, Journal of Approx. Theory 106 (2000), 249-261.

[24] V. A. Skvortsov: On Fourier series with respect to the Walsh-Kaczmarz system, Analysis Math. 7 (1981), 141-150.

[25] A. A. S̆neider: On series with respect to the Walsh functions with monotone coefficients, Izv. Akad. Nauk SSSR Ser. Math. 12 (1948), 179-192.

[26] G.Tephnadze: Fejér means of Vilenkin-Fourier series, Studia. sci. math. Hung., 49 (1), (2012) 79-90.

[27] G.Tephnadze: On the maximal operator of Vilenkin-Fejér means, Turk. J. Math, 37, (2013), 308-318.

[28] G.Tephnadze: On the maximal operators of Vilenkin-Fejér means on Hardy spaces, Mathematical Inequalities \& Applications, 16 (2) (2013), 301-312.

[29] G.Tephnadze: On the maximal operators of Walsh-Kaczmarz-Fejer means, Periodica Mathematica Hungarica, 67, (1), 2013, 33-45.

[30] C. Warari: Best approximation by Walsh polinomials, Tohoku Math. J., 15 (1963), $1-5$

[31] F. Weisz: Martingale Hardy spaces and their applications in Fourier analysis, Springer-Verlang, Berlin, 1994.

[32] F. Weisz: Summability of multi-dimensional Fourier series and Hardy space, Kluwer Academic, Dordrecht, 2002.

[33] F. Weisz: Cesàro summability of one and two-dimensional Walsh-Fourier series, Anal. Math. 22 (1996), 229-242.

[34] F. Weisz: $\theta$-summability of Fourier series, Acta Math. Hungar. 103 (2004), 139-176.

[35] S.H. Yano: Cesáro summability of Walsh-Fourier series, Tohoku Math. J. 15 (1957), 267-272.

[36] W.S. Young: On the a.e converence of Walsh-Kaczmarz-Fourier series, Proc. Amer. Math. Soc. 44 (1974), 353-358.

G. Tephnadze, Department of Mathematics, Faculty of Exact and Natural Sciences, Tbilisi State University, Chavchavadze str. 1, Tbilisi 0128, Georgia and Department of Engineering Sciences and Mathematics, Lule\& University of Technology, SE-971 87, LuleA, Sweden.

E-mail address: giorgitephnadze@gmail.com 